\title{
Effect of exacerbation history on clinical response to dupilumab in moderate-to-severe uncontrolled asthma
}

\author{
Jonathan Corren ${ }^{1}$, Constance H. Katelaris ${ }^{2,3}$, Mario Castro ${ }^{4}$, Jorge F. Maspero ${ }^{5}$, Linda B. Ford ${ }^{6}$, \\ David M.G. Halpin ${ }^{7}$, Megan S. Rice ${ }^{8}$, Amr Radwan $^{9}$, Yamo Deniz ${ }^{9}$, Paul J. Rowe ${ }^{10}$, Ariel Teper ${ }^{10}$ and \\ Michel Djandji ${ }^{8}$
}

${ }^{1}$ David Geffen School of Medicine at UCLA, Los Angeles, CA, USA. ${ }^{2}$ Campbelltown Hospital, Campbelltown, Australia. ${ }^{3}$ Sydney University, Sydney, Australia. ${ }^{4}$ University of Kansas School of Medicine, Kansas City, KS, USA. ${ }^{5}$ Fundación CIDEA, Buenos Aires, Argentina. ${ }^{6}$ The Asthma and Allergy Center, Bellevue, NE, USA. ${ }^{7}$ University of Exeter, Exeter, UK. ${ }^{8}$ Sanofi, Cambridge, MA, USA. ${ }^{9}$ Regeneron Pharmaceuticals, Inc., Tarrytown, NY, USA. ${ }^{10}$ Sanofi, Bridgewater, NJ, USA.

Corresponding author: Jonathan Corren (jcorren@ucla.edu)

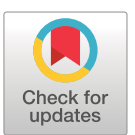

Copyright $\odot$ The authors 2021.

This version is distributed under the terms of the Creative Commons Attribution Non-Commercial Licence 4.0. For commercial reproduction rights and permissions contact permissions@ersnet.org

This article has supplementary material available from erj.ersjournals.com

Received: 11 Dec 2020 Accepted: 16 March 2021

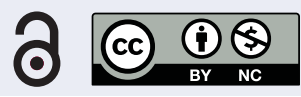

Shareable abstract (@ERSpublications)

Dupilumab reduced severe exacerbations and improved lung function and asthma control in patients with type 2-high asthma, irrespective of exacerbation history and baseline ICS dose. These data will aid clinicians managing patients with severe disease. https://bit.ly/2PjnSm6

Cite this article as: Corren J, Katelaris $\mathrm{CH}$, Castro M, et al. Effect of exacerbation history on clinical response to dupilumab in moderate-to-severe uncontrolled asthma. Eur Respir J 2021; 58: 2004498 [DOI: 10.1183/13993003.04498-2020].

\section{Abstract}

Background The phase 3 LIBERTY ASTHMA QUEST study (ClinicalTrials.gov: NCT02414854) in patients with uncontrolled, moderate-to-severe asthma has demonstrated the efficacy and safety of dupilumab 200 and 300 mg every 2 weeks versus placebo. This post hoc analysis assessed the effect of dupilumab on efficacy outcomes and asthma control across a range of historical exacerbation rates in patients with type 2-high asthma.

Methods Annualised severe exacerbation rates over the 52-week treatment period, pre-bronchodilator forced expiratory volume in $1 \mathrm{~s}\left(\mathrm{FEV}_{1}\right)$ at weeks 12 and 52, and the five-item Asthma Control Questionnaire (ACQ-5) score at weeks 24 and 52 were assessed in patients with $\geqslant 1$, $\geqslant 2$ or $\geqslant 3$ exacerbations in the previous year. Subgroups were stratified by baseline blood eosinophils $\geqslant 150$ or $\geqslant 300$ cells $\mu \mathrm{L}^{-1}$ or baseline exhaled nitric oxide fraction $\geqslant 25 \mathrm{ppb}$ and baseline inhaled corticosteroid (ICS) dose.

Results Across all type 2-high subgroups, dupilumab versus placebo significantly reduced severe exacerbations by $54-90 \%$, with greater improvements in patients with more exacerbations prior to study initiation. Similarly, improvements in $\mathrm{FEV}_{1}$ (least squares (LS) mean difference versus placebo: $\geqslant 1$ exacerbations, $0.15-0.25 \mathrm{~L}$; $\geqslant 2$ exacerbations, 0.12-0.32 L; $\geqslant 3$ exacerbations, 0.09-0.38 L; majority $\mathrm{p}<0.05$ ) and ACQ-5 score (LS mean difference range: $\geqslant 1$ exacerbations, -0.30 to $-0.57 ; \geqslant 2$ exacerbations, -0.29 to -0.56 ; $\geqslant 3$ exacerbations, -0.43 to -0.61 ; all $\mathrm{p}<0.05)$ were observed, irrespective of prior exacerbation history, across all subgroups.

Conclusions Dupilumab significantly reduced severe exacerbations and improved $\mathrm{FEV}_{1}$ and asthma control in patients with elevated type 2 biomarkers irrespective of exacerbation history and baseline ICS dose.

\section{Introduction}

Asthma exacerbations, which may sometimes necessitate a change in a patient's current treatment, account for significant mortality and present a considerable economic burden due to the healthcare costs associated with their management [1, 2]. Patients with type 2 asthma and/or patients receiving higher doses of inhaled corticosteroids (ICS) have more severe disease and require higher doses of steroids to maintain asthma control. These subpopulations are therefore at greater risk of future exacerbations [3]. Moreover, asthma exacerbation history, particularly of recent events, has been shown to be a significant independent predictor of future exacerbation risk $[4,5]$. 
Dupilumab is a fully human VelocImmune-derived monoclonal antibody that blocks the shared receptor component for interleukin (IL)-4 and IL-13, thus inhibiting signalling of both IL-4 and IL-13, which are key and central drivers of type 2 inflammation in multiple diseases [6-9]. These cytokines, together with IL-5, play important roles in the pathogenesis of asthma [9, 10]. Dupilumab is approved for patients aged $\geqslant 12$ years with moderate-to-severe eosinophilic or oral corticosteroid-dependent asthma, for adult patients with inadequately controlled chronic rhinosinusitis with nasal polyps, and for the treatment of patients with inadequately controlled, moderate-to-severe atopic dermatitis aged $>6$ years in the USA and adults in the European Union and other countries [11-20].

In the phase 3 LIBERTY ASTHMA QUEST study, dupilumab 200 and $300 \mathrm{mg}$ every 2 weeks versus matched placebo significantly reduced severe exacerbation rates and improved pre-bronchodilator forced expiratory volume in $1 \mathrm{~s}\left(\mathrm{FEV}_{1}\right)$, asthma control and quality of life measures in patients with uncontrolled, moderate-to-severe asthma [15]. Treatment effects were generally of greater magnitude in patients with elevated baseline levels of type 2 inflammatory biomarkers (blood eosinophils $\geqslant 150$ cells $\mu \mathrm{L}^{-1}$ or exhaled nitric oxide fraction $\left.\left(F_{\mathrm{ENO}}\right) \geqslant 25 \mathrm{ppb}\right)[15]$.

Understanding the efficacy of treatments such as dupilumab in patients with high disease burden (evidenced by frequent, recent exacerbations and high-dose ICS use) is of considerable relevance to clinicians treating patients with severe disease. The aim of this post hoc analysis of the LIBERTY ASTHMA QUEST study was to assess the effect of dupilumab on severe exacerbations, lung function and asthma control in subgroups of patients with a type 2-high phenotype, who had experienced a range of exacerbations in the previous year $(\geqslant 1, \geqslant 2$ or $\geqslant 3)$ and who were further stratified by baseline ICS dose (high versus medium).

\section{Methods}

Study design and patients

LIBERTY ASTHMA QUEST (ClinicalTrials.gov: NCT02414854) was a phase 3 multinational, multicentre, randomised, double-blind, placebo-controlled study that assessed the effect of dupilumab in patients with uncontrolled, moderate-to-severe asthma [15]. Patients aged $\geqslant 12$ years with physician-diagnosed asthma for 12 months (based on the Global Initiative for Asthma 2014 guidelines) were eligible to participate [21]. The study was open to all patients, irrespective of eosinophilic status or any other biomarker requirement. Patients were randomised in a 2:2:1:1 ratio to add-on subcutaneously administered dupilumab $200 \mathrm{mg}$ (loading dose $400 \mathrm{mg}$ ) or $300 \mathrm{mg}$ (loading dose $600 \mathrm{mg}$ ) every 2 weeks or matched-volume placebos for 52 weeks. Full details of the study's design and methodology have been reported previously $[15,22]$; complete patient eligibility criteria, including the requirement for all patients to have had $\geqslant 1$ exacerbations within the year before enrolment, can be found in the supplementary appendix of the primary manuscript [15].

The study was conducted in accordance with the Declaration of Helsinki, the International Conference on Harmonisation Good Clinical Practice guidelines and applicable regulatory requirements. An independent data and safety monitoring committee conducted blinded monitoring of patient safety data. Study conduct and documentation were monitored by local institutional review boards or ethics committees and all patients provided written informed consent before participating in the trial.

\section{Study end-points and populations analysed}

The end-points analysed in this post hoc analysis of LIBERTY ASTHMA QUEST were: annualised rate of severe exacerbations during the 52-week treatment period, least squares (LS) mean change from baseline in pre-bronchodilator $\mathrm{FEV}_{1}$ (L) at weeks 12 and 52, and LS mean change from baseline in the five-item Asthma Control Questionnaire (ACQ-5) score at weeks 24 and 52. A severe asthma exacerbation was defined as a deterioration of asthma requiring treatment with systemic corticosteroids for $>3$ days, or hospitalisation or emergency room visit because of asthma requiring systemic corticosteroids. ACQ-5 is a patient-reported measure of the adequacy of asthma control and of change in asthma control; higher scores indicate less asthma control [23].

Subgroup analyses were performed for patients stratified by number of exacerbations in the previous year $(\geqslant 1, \geqslant 2$ or $\geqslant 3)$, baseline blood eosinophils $\left(\geqslant 150\right.$ or $\geqslant 300$ cells $\left.\mu \mathrm{L}^{-1}\right)$, baseline $F_{\mathrm{ENO}}(\geqslant 25 \mathrm{ppb})$ and baseline ICS dose (medium or high). Analyses were also performed in the subgroup of patients without elevated levels of type 2 biomarkers at baseline (eosinophils $<150$ cells $\mu \mathrm{L}^{-1}$ and $F_{\text {ENO }}<25 \mathrm{ppb}$ ). 


\section{Statistical analysis}

The estimates for adjusted annualised severe exacerbation rates were derived using a negative binomial regression model, with total number of events occurring during the 52-week treatment period as the response variable, and the four intervention groups, age, geographic region, baseline eosinophil strata $(<300$ or $\geqslant 300$ cells $\cdot \mathrm{LL}^{-1}$ ), baseline ICS dose level and number of exacerbations in the previous year as covariates. Change from baseline in pre-bronchodilator $\mathrm{FEV}_{1}$ and ACQ-5 scores was analysed using mixed effects models with repeated measures, including assigned intervention, age, baseline eosinophil strata, visit, intervention-by-visit interaction, region (pooled country), corresponding baseline pre-bronchodilator $\mathrm{FEV}_{1}$ or ACQ-5 value and baseline-by-visit interaction as covariates. Sex and baseline height were included as additional covariates in the models for $\mathrm{FEV}_{1}$. For patients who discontinued the assigned intervention and remained in the trial, measurements after the intervention were discontinued and were included in the analysis.

A p-value of $<0.05$ for the comparison between each dupilumab dose and placebo (within each subgroup) was considered statistically significant.

\section{Results}

\section{Patient characteristics}

1902 patients were enrolled in the LIBERTY ASTHMA QUEST trial (1264 assigned to dupilumab and 638 assigned to placebo), all of whom had experienced $\geqslant 1$ severe exacerbation events in the previous year [15]. There were no major differences in baseline characteristics across the subgroups, although patients who had experienced $\geqslant 3$ exacerbations in the year prior to study entry had relatively lower pre-bronchodilator $\mathrm{FEV}_{1}$ and a higher requirement for ICS use at baseline than did those who had experienced fewer exacerbations. Patients who had experienced $\geqslant 3$ exacerbations also had higher mean baseline levels of blood eosinophils and $F_{\text {ENO }}$ (table 1).

\section{Annualised rate of severe asthma exacerbations}

Dupilumab every 2 weeks (combined treatment groups) significantly reduced severe exacerbation rates versus placebo in patients with elevated baseline blood eosinophils irrespective of exacerbation history or ICS dose at baseline, with a greater magnitude of improvement observed in patients with $\geqslant 2$ or $\geqslant 3$ prior exacerbations (figure 1). Reductions in exacerbation rates versus placebo ranged from $58 \%$ to $71 \%$ in the intention-to-treat (ITT) and $54 \%$ to $65 \%$ in the ICS high-dose subgroup of patients with baseline eosinophils $\geqslant 150$ cells $\cdot \mu \mathrm{L}^{-1}, 66 \%$ to $75 \%$ (ITT) and $63 \%$ to $67 \%$ (high-dose ICS) in patients with baseline eosinophils $\geqslant 300$ cells $\cdot \mu \mathrm{L}^{-1}$, and $63 \%$ to $80 \%$ (ITT) and $62 \%$ to $75 \%$ (high-dose ICS) in patients with baseline $F_{\text {ENO }} \geqslant 25 \mathrm{ppb}$ (all $\mathrm{p}<0.001$ ) (figure $1 \mathrm{a}-\mathrm{c}$ ). Similar trends were observed in patients on medium-dose ICS at baseline (supplementary figure S1a). Across all high type 2 biomarker subgroups, adjusted annualised rates of exacerbation following dupilumab treatment ranged from 0.16 (in patients with $\geqslant 300$ cells $\cdot \mu \mathrm{L}^{-1}$ and medium-dose ICS treated with combined dupilumab 200 and $300 \mathrm{mg}$ ) to 0.65 (patients with $\geqslant 150$ cells $\mu \mathrm{L}^{-1}$ and high-dose ICS treated with combined dupilumab 200 and $300 \mathrm{mg}$ ) compared with rates ranging from 0.86 to 2.35 in placebo-treated patients.

In the subgroup of patients with baseline eosinophils $<150$ cells $\cdot \mu \mathrm{L}^{-1}$ and baseline $F_{\mathrm{ENO}}<25 \mathrm{ppb}$, numerical improvements versus placebo in annualised exacerbation rates were observed, although these were not statistically significant (supplementary figure S1b).

\section{Pre-bronchodilator FEV}

Dupilumab 200 and 300 mg every 2 weeks (combined treatment groups) versus matched placebo improved pre-bronchodilator $\mathrm{FEV}_{1}$ at week 12, irrespective of exacerbation history across all subgroups, stratified by baseline eosinophils, $F_{\text {ENO }}$ or ICS dose (figure 2 and supplementary figure S2); these improvements were statistically significant in a majority of cases and the level of improvement increased with the number of prior exacerbations. In the subpopulation of patients who had elevated baseline biomarker levels (blood eosinophils $\geqslant 150$ or $\geqslant 300$ cells $\cdot \mu \mathrm{L}^{-1}$ or baseline $F_{\mathrm{ENO}} \geqslant 25 \mathrm{ppb}$ ), further stratified by baseline ICS dose groups, and who had experienced $\geqslant 1$ exacerbations in the previous year, LS mean change from baseline at week 12 in pre-bronchodilator $\mathrm{FEV}_{1}$ ranged from 0.35 to $0.46 \mathrm{~L}$ with dupilumab and 0.20 to $0.23 \mathrm{~L}$ with placebo (LS mean difference versus placebo ranged from 0.15 to $0.25 \mathrm{~L}$; all $\mathrm{p}<0.001$ ) (figure $2 \mathrm{a}-\mathrm{c}$, and supplementary figure S2a and b). Improvements with dupilumab were also evident in the elevated biomarker subgroups with $\geqslant 2$ and $\geqslant 3$ prior exacerbations. In the former group, dupilumab treatment increased pre-bronchodilator $\mathrm{FEV}_{1}$ by a range of 0.37 to $0.56 \mathrm{~L}$ compared with changes of 0.19 to $0.28 \mathrm{~L}$ with placebo (LS mean difference versus placebo ranged from 0.12 to $0.31 \mathrm{~L}$; all $\mathrm{p}<0.01$ ) (figure $2 \mathrm{a}-\mathrm{c}$ and supplementary figure S2a). In the subgroups of patients with elevated biomarker levels and $\geqslant 3$ prior exacerbations, improvements with dupilumab and placebo at week 12 were between 0.36 and $0.66 \mathrm{~L}$ and 0.19 and $0.33 \mathrm{~L}$, respectively (LS mean difference versus placebo ranged from 0.09 to $0.38 \mathrm{~L}$; all $\mathrm{p}<0.01$ 
TABLE 1 Baseline demographic and clinical characteristics of LIBERTY ASTHMA QUEST patients by exacerbation history and type 2 biomarkers

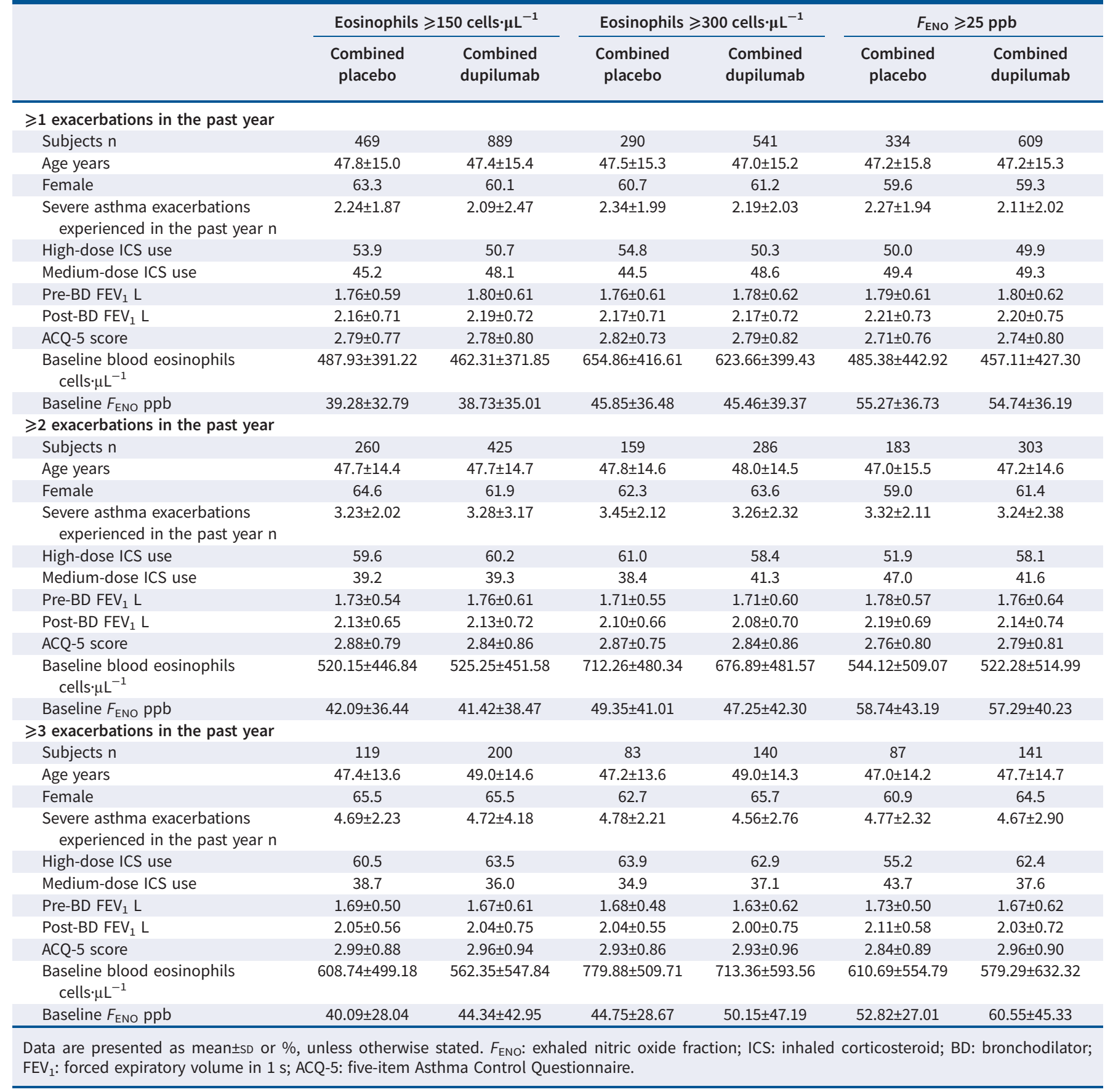

versus placebo except in patients with baseline blood eosinophils $\geqslant 150$ cells $\mu \mathrm{L}^{-1}$ and high ICS dose $(p=0.0679)$ (figure $2 a)$ and patients with baseline blood eosinophils $\geqslant 300$ cells $\mu \mathrm{L}^{-1}$ and high ICS dose ( $p=0.1717$ ) (figure 2b). Improvements were sustained at week 52 (supplementary figure S2a and b).

In the subgroup of patients without elevated baseline biomarkers (i.e. baseline blood eosinophils $<150$ cells $\mu \mathrm{L}^{-1}$ and baseline $F_{\text {ENO }}<25 \mathrm{ppb}$ ), numerical improvements with dupilumab treatment versus placebo were observed at weeks 12 and 52, although these were largely not statistically significant (supplementary figure S2c).

\section{ACQ-5 scores}

Dupilumab every 2 weeks (combined treatment groups) versus matched placebo improved asthma control (ACQ-5 score) at week 24 irrespective of exacerbation history across all subgroups stratified by baseline 


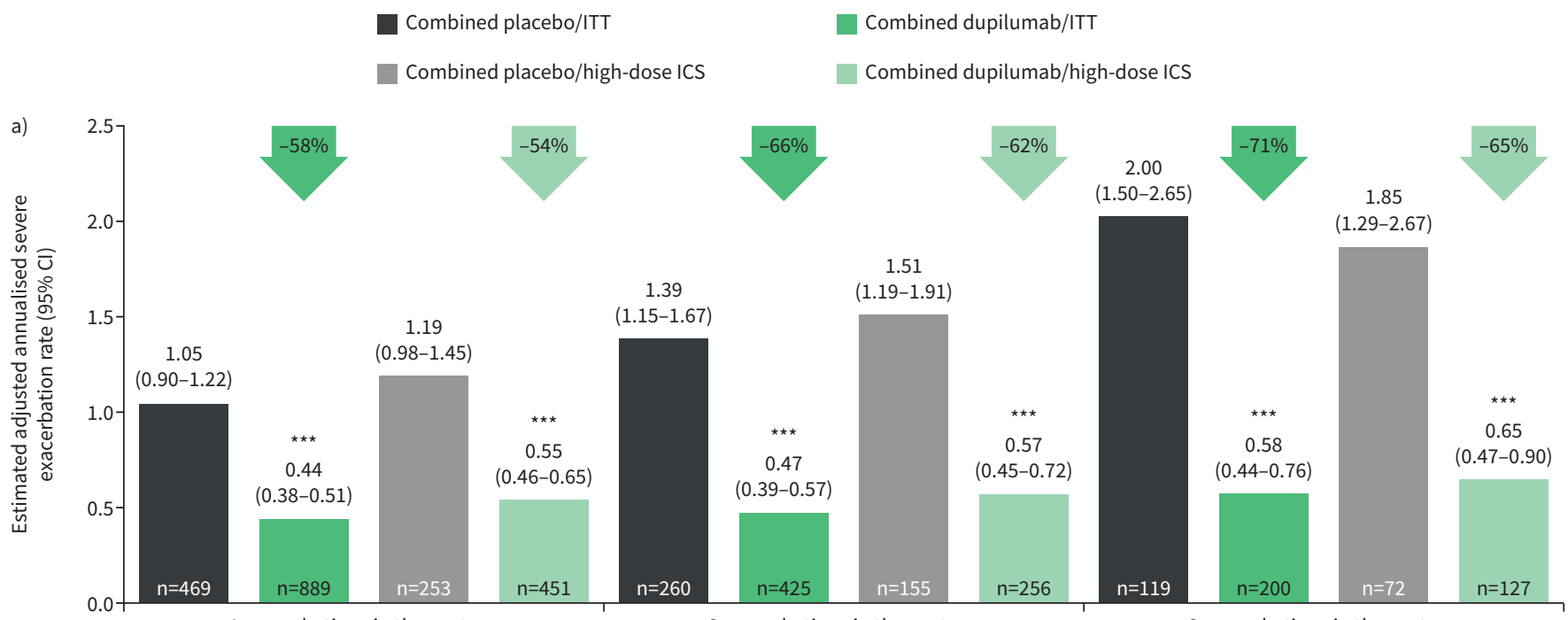

$\geq 1$ exacerbations in the past year

$\geq 2$ exacerbations in the past year

$\geq 3$ exacerbations in the past year
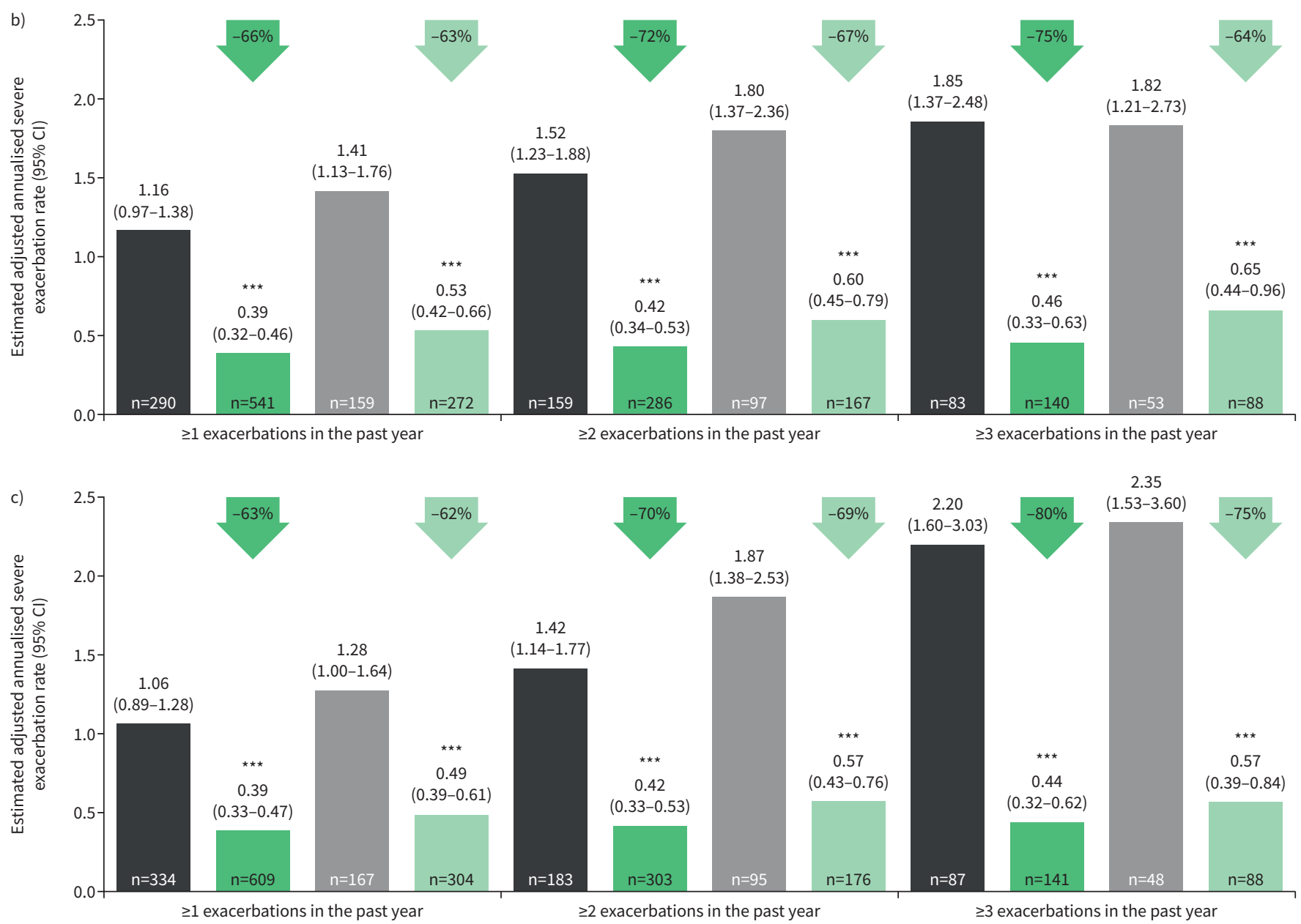

FIGURE 1 Reduction of annualised rate $(95 \% \mathrm{CI})$ of severe exacerbations during the 52-week intervention period in subgroups of LIBERTY ASTHMA QUEST patients categorised by a) baseline blood eosinophils $\geqslant 150$ cells $\mu \mathrm{L}^{-1}$, b) baseline blood eosinophils $\geqslant 300$ cells $\mu \mathrm{L}^{-1}$ and $\mathrm{c}$ ) baseline exhaled nitric oxide fraction $\geqslant 25 \mathrm{ppb}$ (intention-to-treat (ITT) or high-dose inhaled corticosteroid (ICS)). ${ }^{\star \star \star}$ : $\mathrm{p}<0.001$ versus placebo. 
Combined placebo/ITT

Combined placebo/high-dose ICS
Combined dupilumab/ITT

Combined dupilumab/high-dose ICS
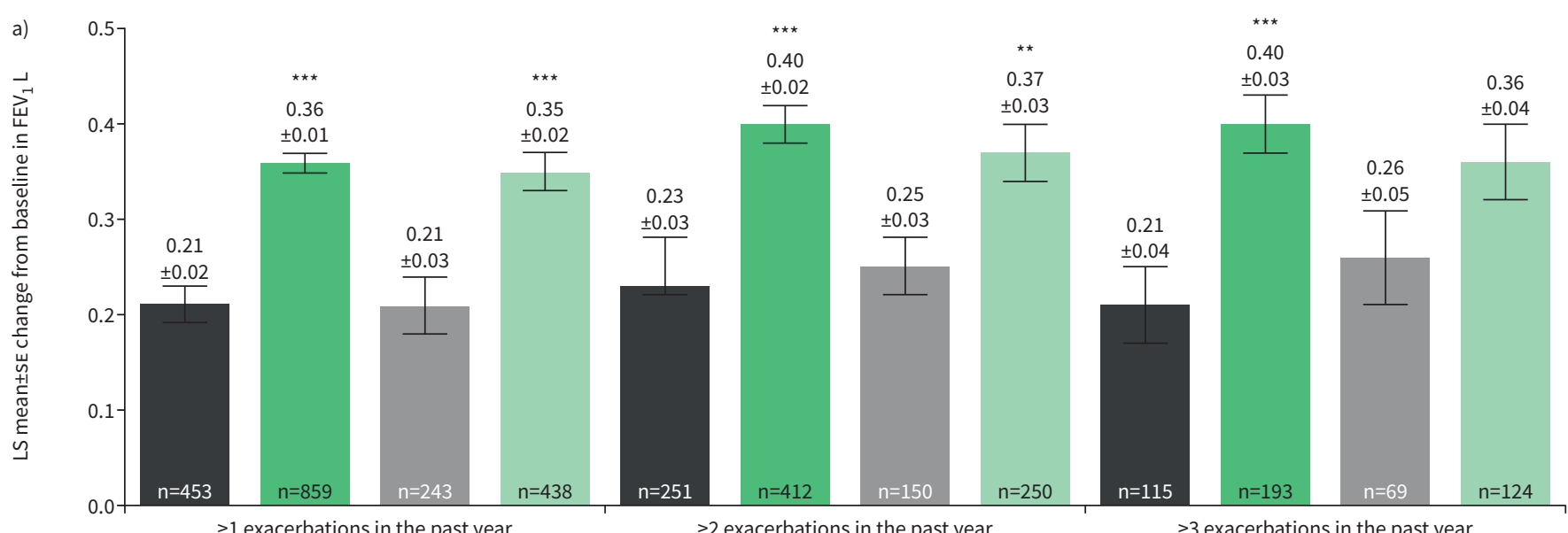

$\geq 1$ exacerbations in the past year

$\geq 2$ exacerbations in the past year
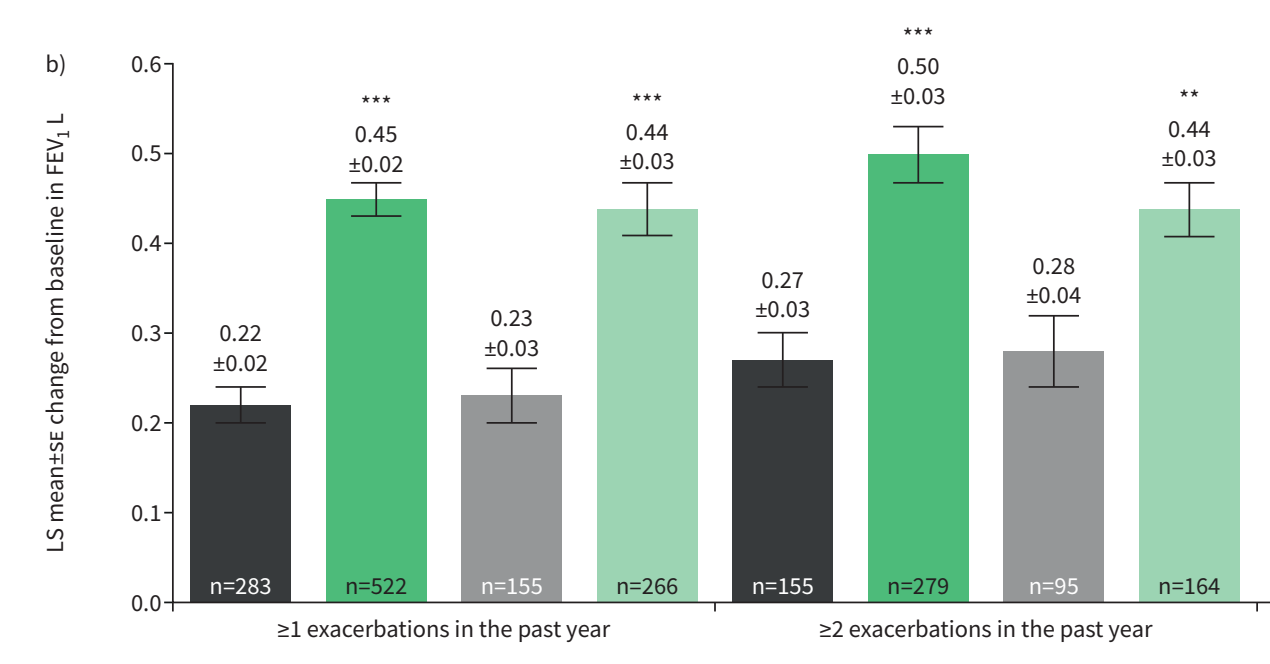

$\geq 3$ exacerbations in the past year
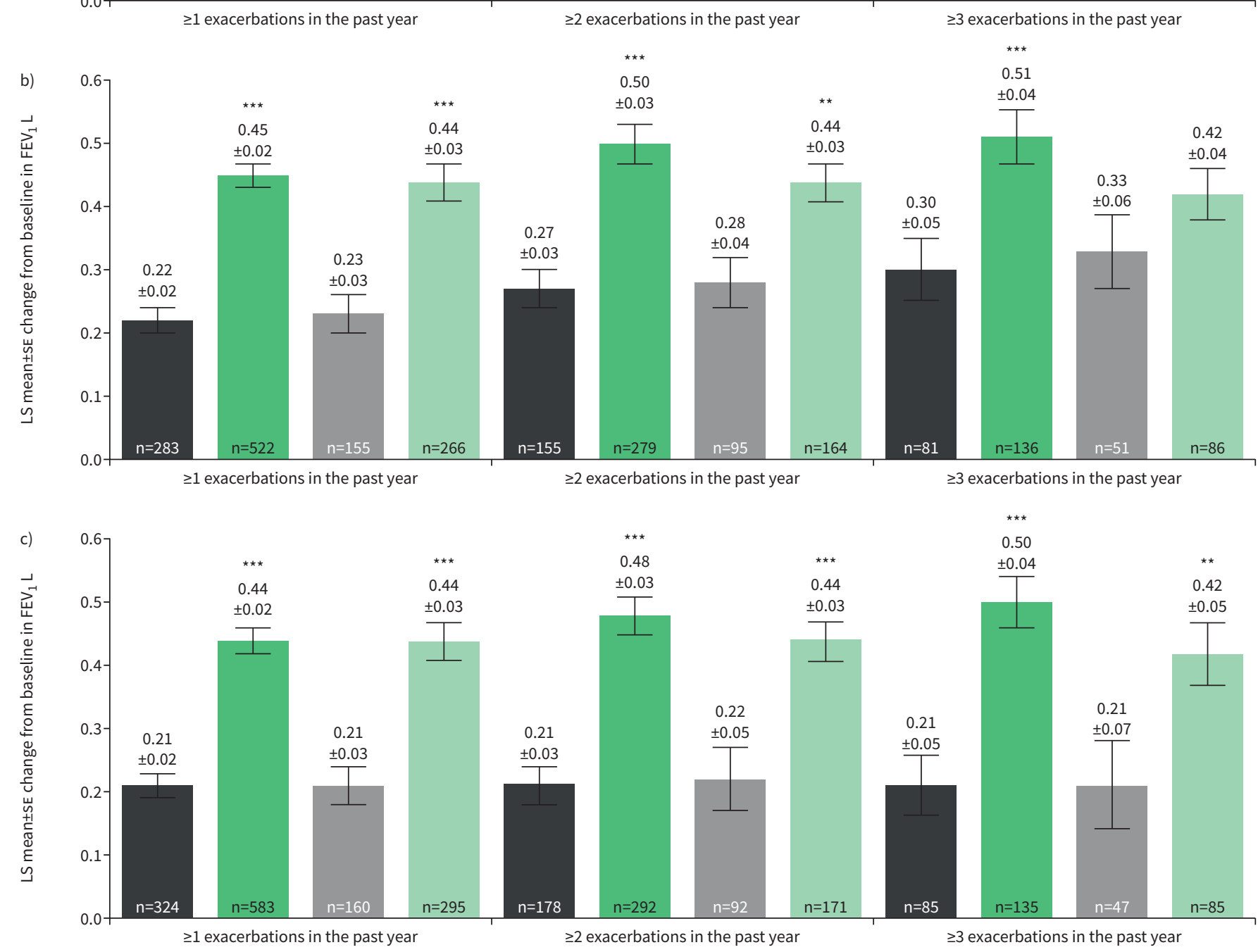

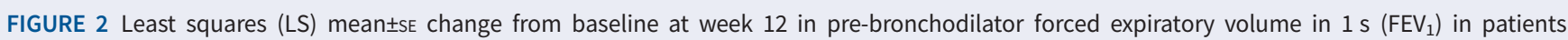
categorised by a) baseline blood eosinophils $\geqslant 150$ cells $\mu \mathrm{L}^{-1}$, b) baseline blood eosinophils $\geqslant 300$ cells $\mu \mathrm{L}^{-1}$ and $\mathrm{c}$ ) baseline exhaled nitric oxide fraction $\geqslant 25 \mathrm{ppb}$ (intention-to-treat (ITT) or high-dose inhaled corticosteroid (ICS)). ${ }^{\star \star}: \mathrm{p}<0.01 ;{ }^{\star \star \star}$ : $\mathrm{p}<0.001$ versus placebo. 
eosinophils, $F_{\text {ENO }}$ or ICS dose (figure 3 and supplementary figure S3). As with the FEV 1 end-point, these improvements versus placebo were statistically significant in a majority of cases, with greater improvements observed in the patients who had elevated biomarker levels at baseline and who had $\geqslant 3$ prior exacerbations. In the subgroups of these patients who had experienced $\geqslant 1$ exacerbations in the previous year, LS mean change in ACQ- 5 score at week 24 ranged from -1.47 to -1.70 for dupilumab and -1.05 to -1.24 for placebo (LS mean difference versus placebo ranged from -0.30 to -0.56 ; all $\mathrm{p}<0.001$ ) (figure $3 \mathrm{a}-\mathrm{c}$, and supplementary figure $\mathrm{S} 3 \mathrm{a}$ and $\mathrm{b}$ ). In those who had $\geqslant 2$ and $\geqslant 3$ exacerbations in the previous year, improvements with dupilumab ranged from -1.51 to -1.83 and -1.58 to -1.90 , respectively; patients receiving placebo reported lower improvements in asthma control, ranging from -1.12 to -1.45 and -1.07 to -1.47 , respectively (LS mean difference versus placebo ranged from -0.29 to -0.56 and -0.43 to -0.61 in patients with $\geqslant 2$ and $\geqslant 3$ prior exacerbations, respectively; all $\mathrm{p}<0.05$ ). Improvements were sustained at week 52 (supplementary figure S3a and b).

As with exacerbation rate and $\mathrm{FEV}_{1}$ end-points, in the subgroup of patients without elevated baseline biomarkers, numerical improvements with dupilumab versus placebo were observed at weeks 24 and 52 (supplementary figure S3c), although these were largely not statistically significant.

\section{Discussion}

This post hoc analysis of the LIBERTY ASTHMA QUEST phase 3, randomised, placebo-controlled trial assessed the effect of add-on dupilumab treatment on annualised rates of severe exacerbations, lung function (pre-bronchodilator $\mathrm{FEV}_{1}$ ) and asthma control (ACQ-5 score) in subgroups of patients categorised by exacerbation history, i.e. $\geqslant 1, \geqslant 2$ or $\geqslant 3$ exacerbations in the year prior to study enrolment. These subpopulations were further stratified by elevated baseline levels of blood eosinophils ( $\geqslant 150$ or $\geqslant 300$ cells $\cdot \mu \mathrm{L}^{-1}$ ) and $F_{\mathrm{ENO}}(\geqslant 25 \mathrm{ppb})$, i.e. two biomarkers of type 2 inflammation, and ICS dose at entry (medium or high). The rationale for selecting these strata was based on the knowledge that recent exacerbations independently predict future exacerbation risk [4, 5], and that patients who have elevated type 2 biomarkers and/or are receiving high-dose ICS have more severe disease and are therefore at greater risk of asthma exacerbations [3, 24]. Indeed, these observations were corroborated by the baseline characteristics of the patients, with the subgroup of patients who had experienced $\geqslant 3$ exacerbations in the year prior to study entry displaying relatively worse lung function (pre-bronchodilator $\mathrm{FEV}_{1}$ ) and a higher requirement for ICS use. Moreover, there also appeared to be a strong link between the number of previous exacerbations experienced and baseline type 2 biomarker levels, indicating that patients with a stronger type 2 signature have higher exacerbation risk.

Across all subgroups, dupilumab 200 and 300 mg every 2 weeks (combined) versus matched placebo significantly reduced severe annualised exacerbation rates, irrespective of the number of exacerbations the patients had experienced in the year prior to the study start. Additionally, there was a trend showing that the magnitude of the improvements compared with placebo increased along with the number of severe asthma exacerbation events experienced in the previous year. Because the risk of exacerbations tends to increase proportionally to the number of recent exacerbations [4, 5], this finding suggests that dupilumab suppresses this increased exacerbation risk, a phenomenon that has also been reported for other asthma biologics [22, 25]. Across all high type 2 biomarker subgroups, even in those considered to be the most severe and difficult to treat due to high rates of prior exacerbations coupled with a high type 2 signature, dupilumab treatment exhibited considerable efficacy in improving lung function, with improvements of up to $0.38 \mathrm{~L}$ compared with placebo. Clinically meaningful improvements were also observed in ACQ-5 score [23], indicating better asthma control, regardless of the number of severe asthma exacerbations patients had experienced in the previous year, or their baseline level of type 2 biomarkers or ICS dose. Even in the most severe patients with a high type 2 signature who had experienced $\geqslant 3$ exacerbations in the previous year, clinically meaningful and statistically significant improvements in ACQ-5 score were observed at week 24 with dupilumab compared with placebo.

For all end-points, numerical improvements with dupilumab compared with placebo treatment were observed in the subgroup of patients who did not display a type 2 phenotype (i.e. who had baseline biomarker concentrations of eosinophils $<150$ cells $\cdot \mu \mathrm{L}^{-1}$ and $F_{\text {ENO }}<25 \mathrm{ppb}$ ). However, most of these improvements were not statistically significant and the magnitude of the treatment effects was minimal compared with those observed in type 2-high subpopulations. That greater treatment effects were observed in patients with elevated type 2 biomarkers is consistent with the mechanism of action of dupilumab, which by binding to IL- 4 receptor $\alpha$ inhibits the type 2 cytokines IL- 4 and IL-13, and thus the type 2 inflammation exhibited by these patients [9]. Similar findings have been reported in other dupilumab asthma studies [14-16]. 
Combined placebo/ITT

Combined placebo/high-dose ICS
Combined dupilumab/ITT

Combined dupilumab/high-dose ICS

a)

$\geq 1$ exacerbations in the past year

$\geq 2$ exacerbations in the past year

$\geq 3$ exacerbations in the past year

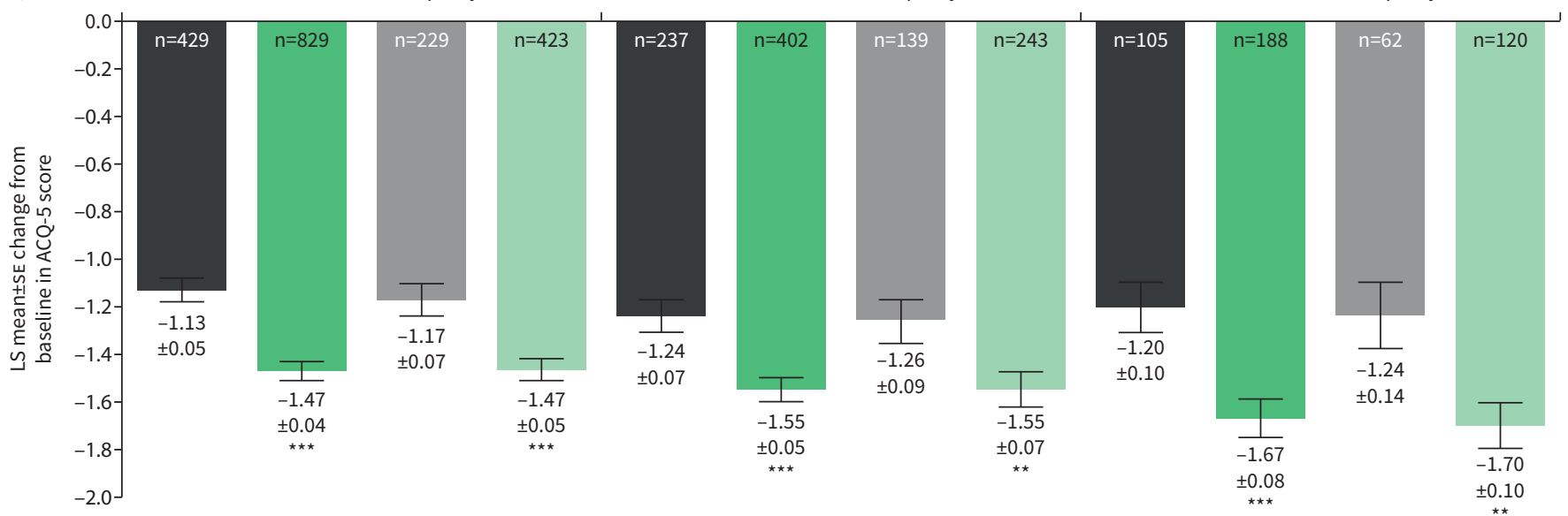

b) $\geq 1$ exacerbations in the past year

$\geq 2$ exacerbations in the past year

$\geq 3$ exacerbations in the past year

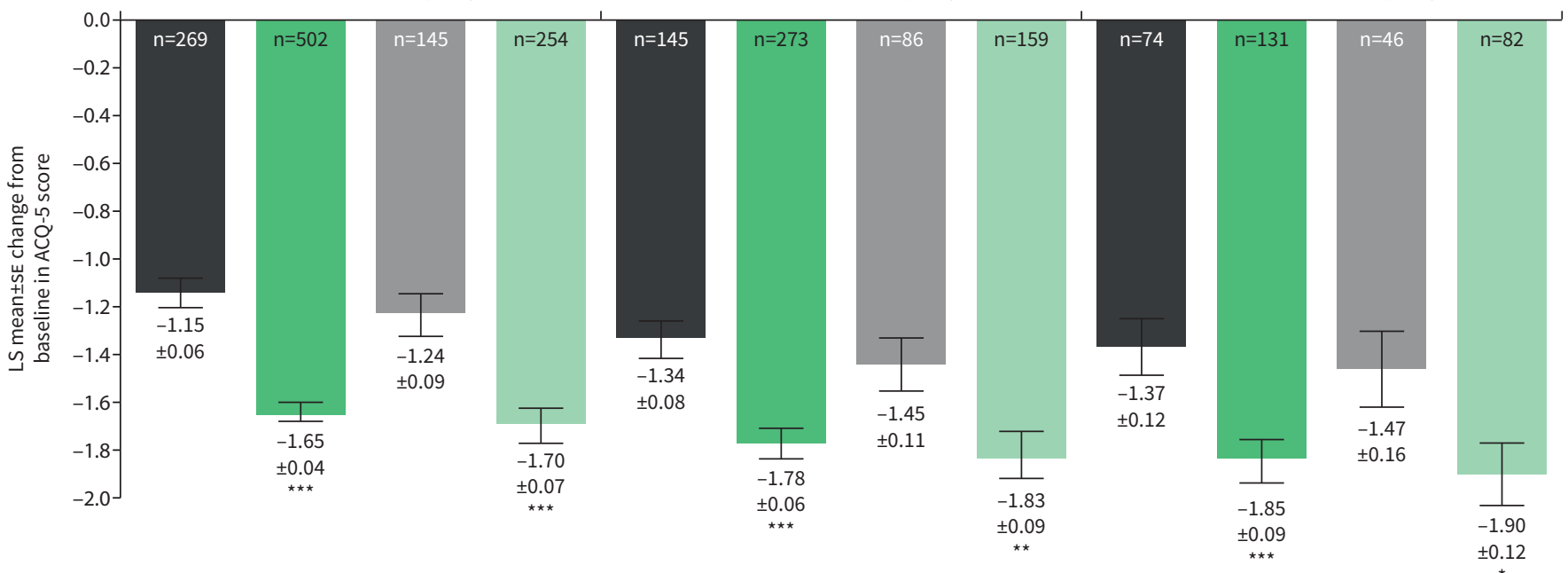

c)

$\geq 1$ exacerbations in the past year

$\geq 2$ exacerbations in the past year

$\geq 3$ exacerbations in the past year

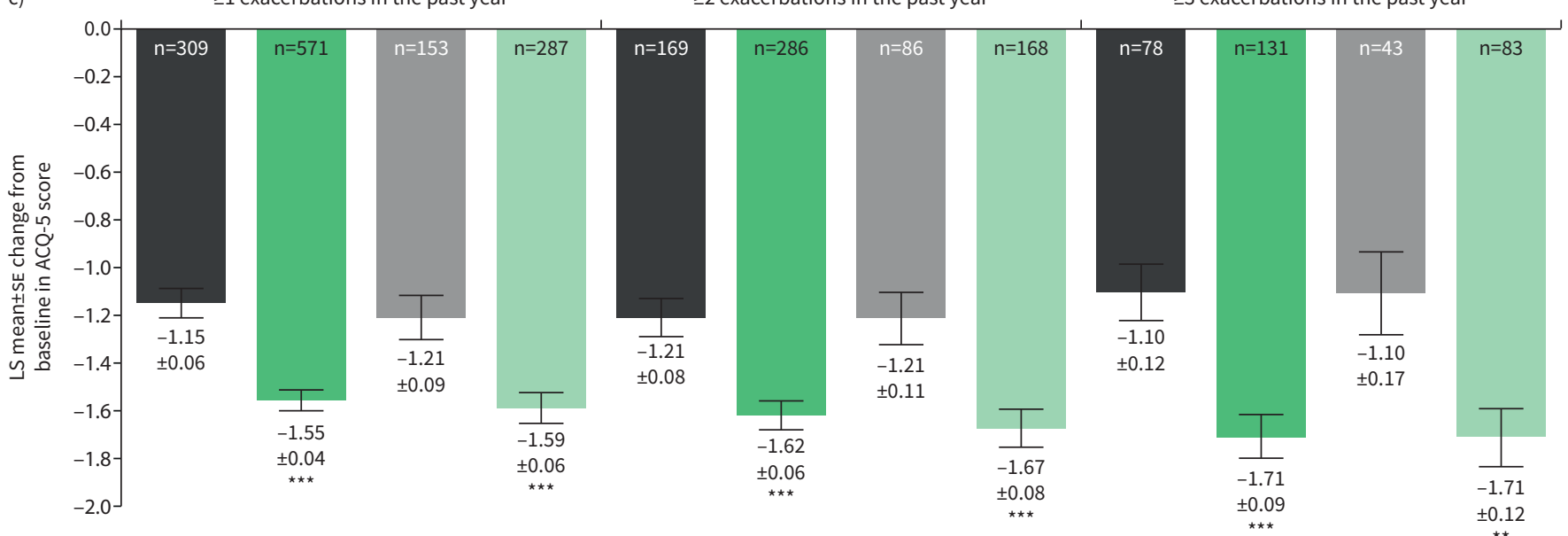

FIGURE 3 Least squares (LS) mean \pm SE change from baseline at week 24 in five-item Asthma Control Questionnaire (ACQ-5) score in patients categorised by a) baseline blood eosinophils $\geqslant 150$ cells $\mu \mathrm{L}^{-1}$, b) baseline blood eosinophils $\geqslant 300 \mathrm{cells} \mu \mathrm{L}^{-1}$ and $\mathrm{c}$ ) baseline exhaled nitric oxide fraction $\geqslant 25 \mathrm{ppb}$ (intention-to-treat (ITT) or high-dose inhaled corticosteroid (ICS)). *: $p<0.05 ;{ }^{\star \star}$ : $p<0.01 ;{ }^{\star \star \star}$ : $p<0.001$ versus placebo. 
Although the data analysed in this study were collected in a large, randomised, stringently controlled clinical trial, a limitation of the analysis presented is its post hoc nature, as the parent study was not powered to specifically investigate differences in the subpopulations described. Accordingly, sample sizes for many of the subgroups analysed and described were low. Additionally, the aetiology of many of the exacerbations assessed in this study, whether in the year prior to study start or during the study itself, were unknown. Exacerbations can be triggered by multiple factors, including viral or bacterial respiratory infection, environmental allergens, pollutants and occupational exposures [26]. With hindsight, better characterisation of the causes of the exacerbations studied could have facilitated a comparative examination of dupilumab efficacy in each of these phenotypes.

In conclusion, this post hoc analysis confirms that the significant reduction in severe exacerbation rates and improvements in asthma control and lung function observed with dupilumab in the overall ITT population of LIBERTY ASTHMA QUEST extend to patients with elevated type 2 inflammation biomarkers, irrespective of exacerbation history and ICS dose at baseline. These findings suggest that prior history of exacerbations adds no further value to prognostication for the treating clinician but is of considerable value to clinicians in order to understand the efficacy of dupilumab, particularly among patients with a very severe disease burden.

Acknowledgements: We thank Nora Crikelair (Regeneron Pharmaceuticals, Inc., USA) and Colin Mitchell (Sanofi, USA) for their contributions. Medical writing/editorial assistance was provided by Sinéad Holland (Excerpta Medica, UK).

LIBERTY ASTHMA QUEST is registered at ClinicalTrials.gov with identifier number NCT02414854. Qualified researchers may request access to patient-level data and related study documents including the clinical study report, study protocol with any amendments, blank case report form, statistical analysis plan and dataset specifications. Patient-level data will be anonymised and study documents will be redacted to protect the privacy of our trial participants. Further details on Sanofi's data sharing criteria, eligible studies and process for requesting access can be found at: http://www.clinicalstudydatarequest.com.

Conflict of interest: J. Corren reports nonfinancial support for research from Sanofi, outside the submitted work. C.H. Katelaris reports nonfinancial support (advisory board member and principal study investigator) from Sanofi, outside the submitted work. M. Castro reports nonfinancial support for research from the American Lung Association, Gossamer, NIH, PCORI and Shionogi, personal fees for lectures and nonfinancial support for research from AstraZeneca, personal fees for lectures and consultancy, and nonfinancial support for research from Boehringer Ingelheim and Sanofi, personal fees for consultancy and nonfinancial support for research from Novartis, personal fees for consultancy from Boston Scientific and Vida Pharma, personal fees for lectures and consultancy from Teva, personal fees for lectures from Genentech and Regeneron Pharmaceuticals, Inc., personal fees (royalties) from Elsevier, outside the submitted work. J.F. Maspero reports being a speaker for AstraZeneca, GlaxoSmithKline, Menarini, Novartis, Sanofi, Teva and Uriach; and has received research grants from Novartis, outside the submitted work. L.B. Ford reports nonfinancial support for research from AstraZeneca, DBV, Genentech, Gossamer, Novartis and Teva, personal fees for consultancy from Sanofi, outside the submitted work. D.M.G. Halpin reports personal fees for lectures and advisory board work from AstraZeneca, Boehringer Ingelheim, Chiesi, CSL Behring, GlaxoSmithKline, Novartis, Pfizer, Sandoz and Sanofi, outside the submitted work. M.S. Rice is an employee of Sanofi and may hold stock and/or stock options in the company. A. Radwan is an employee and shareholder of Regeneron Pharmaceuticals, Inc. Y. Deniz is an employee and shareholder of Regeneron Pharmaceuticals, Inc. P.J. Rowe is an employee of Sanofi and may hold stock and/or stock options in the company. A. Teper is a former employee of Sanofi. M. Djandji is an employee of Sanofi and may hold stock and/or stock options in the company.

Support statement: Research sponsored by Sanofi and Regeneron Pharmaceuticals, Inc. Medical writing/editorial assistance was funded by Sanofi Genzyme and Regeneron Pharmaceuticals, Inc. Funding information for this article has been deposited with the Crossref Funder Registry.

\section{References}

1 Krishnan V, Diette GB, Rand CS, et al. Mortality in patients hospitalized for asthma exacerbations in the United States. Am J Respir Crit Care Med 2006; 174: 633-638.

2 Lane S, Molina J, Plusa T. An international observational prospective study to determine the cost of asthma exacerbations (COAX). Respir Med 2006; 100: 434-450.

3 Fahy JV. Type 2 inflammation in asthma - present in most, absent in many. Nat Rev Immunol 2015; 15 : 57-65. 
4 Miller MK, Lee JH, Miller DP, et al. Recent asthma exacerbations: a key predictor of future exacerbations. Respir Med 2007; 101: 481-489.

5 Tanaka $\mathrm{A}$, Uno $\mathrm{T}$, Sato $\mathrm{H}$, et al. Predicting future risk of exacerbations in Japanese patients with adult asthma: a prospective 1-year follow-up study. Allergol Int 2017; 66: 568-573.

6 Macdonald LE, Karow M, Stevens S, et al. Precise and in situ genetic humanization of $6 \mathrm{Mb}$ of mouse immunoglobulin genes. Proc Natl Acad Sci USA 2014; 111: 5147-5152.

7 Murphy AJ, Macdonald LE, Stevens S, et al. Mice with megabase humanisation of their immunoglobulin genes generate antibodies as efficiently as normal mice. Proc Natl Acad Sci USA 2014; 111: 5153-5158.

8 Gandhi NA, Pirozzi G, Graham NMH. Commonality of the IL-4/IL-13 pathway in atopic diseases. Expert Rev Clin Immunol 2017; 13: 425-437.

9 Le Floc'h A, Allinne J, Nagashima K, et al. Dual blockade of IL-4 and IL-13 with dupilumab, an IL-4Ro antibody, is required to broadly inhibit type 2 inflammation. Allergy 2020; 75: 1188-1204.

10 Locksley RM. Asthma and allergic inflammation. Cell 2010; 140: 777-783.

11 Regeneron Pharmaceuticals, Inc. DUPIXENT (dupilumab) injection, for subcutaneous use. Prescribing information. 2017. www.regeneron.com/sites/default/files/Dupixent_FPI.pdf Date last accessed: 1 October 2020.

12 Sanofi-Aventis Groupe. DUPIXENT (dupilumab) $300 \mathrm{mg}$ solution for injection in pre-filled syringe. Summary of product characteristics. 2017. https://ec.europa.eu/health/documents/community-register/2019/201905061445 41/anx_144541_en.pdf Date last accessed: 1 October 2020.

13 Pharmaceuticals and Medical Devices Agency. DUPIXENT (dupilumab). 2017. www.pmda.go.jp/files/000235351.pdf Date last accessed: 1 September 2020.

14 Wenzel S, Castro M, Corren J, et al. Dupilumab efficacy and safety in adults with uncontrolled persistent asthma despite use of medium-to-high-dose inhaled corticosteroids plus a long-acting $\beta 2$ agonist: a randomised double-blind placebo-controlled pivotal phase 2b dose-ranging trial. Lancet 2016; 388: 31-44.

15 Castro M, Corren J, Pavord ID, et al. Dupilumab efficacy and safety in moderate-to-severe uncontrolled asthma. N Engl J Med 2018; 378: 2486-2496.

16 Rabe KF, Nair P, Brusselle G, et al. Efficacy and safety of dupilumab in glucocorticoid-dependent severe asthma. N Engl J Med 2018; 378: 2475-2485.

17 Bachert C, Han JK, Desrosiers M, et al. Efficacy and safety of dupilumab in patients with severe chronic rhinosinusitis with nasal polyps (LIBERTY NP SINUS-24 and LIBERTY NP SINUS-52): results from two multicentre, randomised, double-blind, placebo-controlled, parallel-group phase 3 trials. Lancet 2019; 394: 1638-1650.

18 Blauvelt A, de Bruin-Weller M, Gooderham M, et al. Long-term management of moderate-to-severe atopic dermatitis with dupilumab and concomitant topical corticosteroids (LIBERTY AD CHRONOS): a 1-year, randomised, double-blinded, placebo-controlled, phase 3 trial. Lancet 2017; 389: 2287-2303.

19 Simpson EL, Bieber T, Guttman-Yassky E, et al. Two phase 3 trials of dupilumab versus placebo in atopic dermatitis. N Engl J Med 2016; 375: 2335-2348.

20 Thaçi D, Simpson EL, Beck LA, et al. Efficacy and safety of dupilumab in adults with moderate-to-severe atopic dermatitis inadequately controlled by topical treatments: a randomised, placebo-controlled, dose-ranging phase $2 \mathrm{~b}$ trial. Lancet 2016; 387: 40-52.

21 Global Initiative for Asthma (GINA). Global Strategy for Asthma Management and Prevention. 2015. Available from: http://ginasthma.org/

22 Busse WW, Maspero JF, Rabe KF, et al. Liberty Asthma QUEST: phase 3 randomised, double-blind, placebo-controlled, parallel-group study to evaluate dupilumab efficacy/safety in patients with uncontrolled, moderate-to-severe asthma. Adv Ther 2018; 35: 737-748.

23 Juniper EF, Svensson K, Mörk AC, et al. Measurement properties and interpretation of three shortened versions of the asthma control questionnaire. Respir Med 2005; 99: 553-558.

24 O'Byrne P, Fabbri LM, Pavord ID, et al. Asthma progression and mortality: the role of inhaled corticosteroids. Eur Respir J 2019; 54: 1900491.

25 Edris A, De Feyter S, Maes T, et al. Monoclonal antibodies in type 2 asthma: a systematic review and network meta-analysis. Respir Res 2019; 20: 179.

26 Singh AM, Busse WW. Asthma exacerbations. 2: aetiology. Thorax 2006; 61: 809-816. 